\title{
Explaining the MiniBooNE excess through a mixed model of neutrino oscillation and decay
}

\author{
S. Vergani, ${ }^{1, *}$ N. W. Kamp, ${ }^{2, \dagger}$ A. Diaz, ${ }^{2, *}$ C. A. Argüelles $\odot^{3, \$}$ J. M. Conrad ${ }^{2, \|}$ M. H. Shaevitz, ${ }^{4, \pi}$ and M. A. Uchida $\odot^{1, * *}$ \\ ${ }^{1}$ University of Cambridge, Cambridge CB3 OHE, United Kingdom \\ ${ }^{2}$ Department of Physics, Massachusetts Institute of Technology, Cambridge, Massachusetts 02139, USA \\ ${ }^{3}$ Department of Physics, Harvard University, Cambridge, Massachusetts 02138, USA \\ ${ }^{4}$ Department of Physics, Columbia University, New York, New York 10027, USA
}

(Received 26 May 2021; accepted 29 September 2021; published 8 November 2021)

\begin{abstract}
The electronlike excess observed by the MiniBooNE experiment is explained with a model comprising a new low mass state $(\mathcal{O}(1) \mathrm{eV})$ participating in neutrino oscillations and a new high mass state $(\mathcal{O}(100) \mathrm{MeV})$ that decays to $\nu+\gamma$. Short-baseline oscillation datasets are used to predict the oscillation parameters. Fitting the MiniBooNE energy and scattering angle data, there is a narrow joint allowed region for the decay contribution at $95 \% \mathrm{CL}$. The result is a substantial improvement over the single sterile neutrino oscillation model, with $\Delta \chi^{2} /$ dof $=19.3 / 2$ for a decay coupling of $2.8 \times 10^{-7} \mathrm{GeV}^{-1}$, high mass state of $376 \mathrm{MeV}$, oscillation mixing angle of $7 \times 10^{-4}$ and mass splitting of $1.3 \mathrm{eV}^{2}$. This model predicts that no clear oscillation signature will be observed in the FNAL short baseline program due to the low signal-level.
\end{abstract}

DOI: 10.1103/PhysRevD.104.095005

\section{INTRODUCTION}

For the past 25 years, anomalies have been observed in short-baseline (SBL) neutrino oscillation experiments. These have been studied under a model called " $3+1$ " that introduces a new non-interacting, hence "sterile," state with mass of $\mathcal{O}(1 \mathrm{eV})$, in addition to the three Standard Model (SM) neutrino states. In this model, $\nu_{\mu} \rightarrow \nu_{e}$ appearance, $\nu_{e}$ disappearance, and $\nu_{\mu}$ disappearance searches should all point to neutrino oscillations at $L / E \sim 1 \mathrm{~m} / \mathrm{MeV}$, where $L$ is the distance a neutrino of energy $E$ travels, with a consistent set of flavor mixing parameters [1-4]. However, while individually the data appear to fit oscillations, global fits find a small probability that all of the relevant datasets are explained by the same parameters [2,3], as measured by the parameter goodness of fit (PGF) test $[5,6]$. In particular, appearance data from MiniBooNE produces large tension between appearance and disappearance in the $3+1$ model. This is because the $3+1$ best-fit parameters from the other

\footnotetext{
*sv408@hep.phy.cam.ac.uk nwkamp@mit.edu *diaza@mit.edu

\$carguelles@fas.harvard.edu

"conrad@mit.edu

"shaevitz@nevis.columbia.edu

mauchida@hep.phy.cam.ac.uk
}

Published by the American Physical Society under the terms of the Creative Commons Attribution 4.0 International license. Further distribution of this work must maintain attribution to the author(s) and the published article's title, journal citation, and DOI. Funded by SCOAP ${ }^{3}$. datasets yield a poor fit to the lowest energy range of the MiniBooNE anomaly [7]. Therefore, there is significant interest in explanations for MiniBooNE beyond the $3+1$ model; for example, one can consider decays of a sterile neutrinos into active neutrinos and singlet scalars $[8,9]$.

The MiniBooNE anomaly is a $4.8 \sigma$ excess of electronlike events observed in interactions from a predominantly muon neutrino beam in a Cherenkov detector [10], which cannot distinguish between electromagnetic showers from electrons and photons. Hence, a favored alternative to the $3+1$ model has been to introduce $\mathrm{MeV}$-scale heavy neutral leptons (HNLs) that decay via $\mathcal{N} \rightarrow \nu \gamma$ within the detector, where the photon is then misidentified as an electron [11-18]; see Refs. [19-27] for misidentified di-electron scenarios. These initial studies of $\mathcal{N}$-decay models describe the MiniBooNE energy distribution well but omit the $3+1$ oscillations predicted from fits to the other anomalies.

In this work, we explore a combination of the two explanations by fitting the MiniBooNE energy and angle distributions using a combined model, $3+1+\mathcal{N}$-decay. The $3+1$ oscillation component has been obtained by fitting SBL datasets other than MiniBooNE appearance. We will show that such a model explains the data well, identifying a highly limited range for the four model parameters: the mixing angle, $\sin ^{2} 2 \theta$, and mass splitting, $\Delta m^{2}$, for the oscillation; and the HNL mass, $m_{\mathcal{N}}$, and photon coupling, $d$, for the decay.

\section{MODEL}

The combination of eV-scale and $\mathrm{MeV}$-scale mass states is motivated if the two are members of a family of $\mathcal{N}_{j}$ 
where $j=1,2,3$. If the mass splittings are similar to the quark and charged-lepton sectors, then the family might also include a keV-scale member [28,29]. All members may interact with photons at a weak level through a dipole portal interaction [17], also known as neutrino magnetic moment [30-35]. Thus, the $\mathcal{N}_{1}=\nu_{4}$ can decay, but the lifetime is typically longer than the age of the Universe [31,36]. The $\mathrm{keV}$-scale mass state, $\mathcal{N}_{2}$, would have a lifetime that is too long to be observed through decay in terrestrial experiments but could explain observed x-ray lines [28,37]. Only the $\mathcal{N} \equiv \mathcal{N}_{3}$ would decay on length scales relevant to SBL experiments. Conversely, only the eV-scale mass state would have sufficiently small mass splitting with respect to the light neutrino states [38-41] to form observable oscillations. In the $3+1+\mathcal{N}$-decay model, any given SBL experiment may be sensitive to signatures of $3+1$ oscillations only, $\mathcal{N} \rightarrow \nu \gamma$ only, or both.

In our model, the production and decay of $\mathcal{N}_{j}$ is due to a dipole portal interaction between left-handed neutrinos, photons, and right-handed HNLs. The $\mathcal{N}_{j}$ are added to the SM Lagrangian using the following term $[15,17]$ :

$$
\begin{aligned}
\mathcal{L} \supset & \mathcal{L}_{\mathrm{SM}}+\sum_{j=1}^{3}\left[\overline{\mathcal{N}}_{j}\left(i \not \partial-M_{j}\right) \mathcal{N}_{j}\right. \\
& \left.+\sum_{\alpha \in\{e, \mu, \tau\}}\left(d_{\alpha j} \bar{\nu}_{i} \sigma_{\mu \nu} F^{\mu \nu} \mathcal{N}_{j}+\text { H.c. }\right)\right],
\end{aligned}
$$

where the $\nu_{i}$ correspond to the light neutrino mass states and $F^{\mu \nu}$ is the electromagnetic field strength. The dimension-full $d_{\alpha j}$ couplings control the strength of the electromagnetic interactions between neutrino species, namely the strength of process like $\mathcal{N}_{j} \rightarrow \nu_{i} \gamma$. Note that $d_{\alpha j}$ reflects the effective dipole coupling of $\mathcal{N}_{j}$ to the weak eigenstate $\nu_{\alpha}$.

This leads to two production mechanisms for $\mathcal{N}_{j}$ : coupling to virtual photons produced in meson decays, such as $\pi^{0}$, and Primakoff upscattering of active neutrinos to $\mathcal{N}$ as they traverse material. Feynman diagrams for the two production (left, middle) and decay (right) processes are shown in Fig. 1.

In our analysis, we considered only production and decay of the third mass state $\mathcal{N}$. This follows if $d_{\alpha j}$ is the same for all generations and is found to be sufficiently small, such that upscattering is rare, because then the small masses of states 1 and 2 lead to lifetimes that are too long for an SBL experiment to observe decay. In this case, we

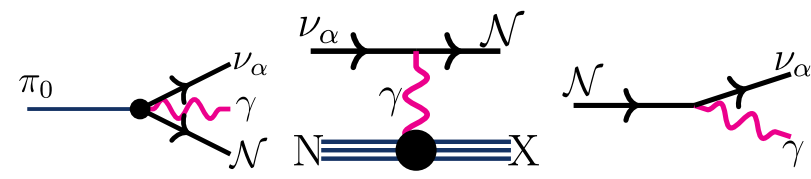

FIG. 1. $\mathcal{N}$ production from $\pi^{0}$ Dalitz decay (left) and $\nu$ upscattering (middle), and decay (right). define $d \equiv d_{\alpha j}$ as a universal coupling. An alternative explanation if $d$ is found to be large is that the $d_{\alpha j}$ vary with family member, suppressing decays of the first and second mass states. In this case, we define $d \equiv d_{\alpha 3}$, where the coupling of $\mathcal{N}_{3}$ to all light neutrino species is assumed to be the same. In the decay, the polarization of $\mathcal{N}$ must be considered [42-44]. The photon from a right-handed Dirac $\mathcal{N}$ decay has a $(1-\cos \theta)$ distribution, where $\theta$ is the angle between the $\mathcal{N}$ and photon momentum vectors; conversely, a left-handed Dirac $\mathcal{N}$ will decay with a $(1+\cos \theta)$ distribution for the photons. Production through an unpolarized virtual, off-shell photon yields an equal combination of right-handed and left-handed $\mathcal{N}$, leading to an effectively isotropic photon decay distribution. For the case of upscattering, where the $\mathcal{N}$ is produced from an interaction with a left-handed muon neutrino, the outgoing $\mathcal{N}$ will be right-handed and the $(1-\cos \theta)$ angular distribution of the photons must be considered. All three new mass states are related via a mixing matrix to the flavor states. Calling the new sterile flavors $s_{j}$, the mass and flavor states are related by:

$\nu_{\alpha}=\sum_{i} U_{\alpha i} \nu_{j}+U_{\alpha 3+i} \mathcal{N}_{i},\left(\alpha=e, \mu, \tau ; s_{1}, s_{2}, s_{3}\right)$,

where $U_{\alpha j}$ is the extended $6 \times 6$ neutral-lepton mixing matrix [1].

\section{CONSTRAINTS}

Three SBL experiments have relevant limits to $\mathcal{N}$ with mass $>10 \mathrm{MeV}$. While not appearing directly in the fit, the viable solution must fall outside of these limits. NOMAD and CHARM-II were high-energy neutrino experiments with too small $L / E$ to be sensitive to the $3+1$ parameters under discussion. The NOMAD analysis searched directly for photons from HNL decay [14]. CHARM-II could not differentiate electrons from photons, and the limit is derived from comparing $\nu_{\mu}$-electron elastic scattering (ES) data to the SM prediction [45]. At larger $\mathcal{N}$ masses, which are kinematically inaccessible in the former process, contributions from $\nu_{\mu}$-nucleon upscattering are also present [22]. However, a detailed analysis of this process in CHARM-II has not yet been performed. LSND has also released $\nu_{\mu}$ electron scattering results in agreement with the SM, placing a limit on $\mathcal{N}$ [17].

Cosmological observations place constraints on additional neutrino species. In order to alleviate tension with a light sterile neutrino [46-52], one can invoke either noncanonical cosmological scenarios [53,54] or secret neutrino interactions [55-65]. HNL interactions similar to those studied in this model also play an important role in cosmology [66], where they may impact big bang nucleosynthesis, relax cosmological bounds on neutrino masses [67], or explain the Hubble tension [68]. 


\section{OSCILLATION GLOBAL FIT}

If the maximum energy of the neutrino source is too small to produce the heavier $\mathcal{N}$ state, then the SBL experiments can observe only $3+1$ oscillations. We refer to this collection of SBL experiments that are not sensitive to $\mathcal{N}$ decay as " $3+1$-only" experiments. The references for relevant " $3+1$-only" experiments used in this analysis are provided in Table I (top), including experiments with anomalies of significance from $2 \sigma$ to $4.8 \sigma$ and experiments consistent with $\nu \mathrm{SM}$ oscillations. The experiments, individually listed, can also be found in Supplemental Material [69] Table II. We use these experiments to determine the oscillation parameters $\sin ^{2}(2 \theta)$ and $\Delta m^{2}$ in a $3+1$-only fit. Notably, the " $3+1$-only" experiments exclude all MiniBooNE results, as we will then use MiniBooNE to fit for the $\mathcal{N}$ parameters $m_{\mathcal{N}}$ and $d$.

As shown in Table I (bottom), we used MiniBooNE data to fit for the $\mathcal{N}$ parameters $m_{\mathcal{N}}$ and $d$, given the oscillation parameters from the $3+1$-only fit. MiniBooNE has excesses in three appearance data subsets: neutrino-mode [88], antineutrino mode [89], and with an off-axis beam [90]. All three cases are compatible with either $3+1$ or HNL explanations. However, the latter two running modes had low statistics and more limited data releases, so we restricted our fit to the neutrino-mode sample.

For the $3+1$-only fit, mixing between heavy neutrinos and the three lightest mass states is constrained to be small by terrestrial measurements at accelerators [91]. Further, oscillations involving the two largest mass states do not contribute to the explaining the anomalies considered in this work.Therefore, we have explicitly assumed no mixing between the two largest mass states and the active states. The only relevant squared-mass-splitting $\Delta m^{2}$ is between the lightest mostly sterile and the mostly active states, where the masses of the latter are assumed to be degenerate and negligible. We concentrated on the $4 \times 4$ neutrallepton-mixing submatrix that relates the lightest mass states to their flavor states. For $U_{\alpha k}$, where $\alpha$ is the flavor and $k$ is the mass state, the mixing angles for the appearance and disappearance oscillation signatures are not independent:

TABLE I. Datasets used in this paper. These include reactor, radioactive, decay-at-rest (DAR) and decay-in-flight (DIF) neutrino sources. For a detailed overview of the datasets used in each of the fits see Supplemental Table II.

\begin{tabular}{lcc}
\hline \hline Used to Test & References (Flux Type) & Type of Fit \\
\hline $\bar{\nu}_{e}$ disappearance & {$[70-74]$ (Reactor) } & \\
$\nu_{e}$ disappearance & {$[75-77]$ (Source) } & \\
$\bar{\nu}_{\mu} \rightarrow \bar{\nu}_{e}$ appearance & {$[78,79](\pi / \mu$ DAR) } & $\uparrow$ \\
$\nu_{\mu} \rightarrow \nu_{e}$ appearance & {$[80](\pi / \mu$ DIF) } & $3+1$-only \\
$\bar{\nu}_{\mu}$ disappearance & {$[81-84](\pi / \mu$ DIF) } & $\downarrow$ \\
$\nu_{\mu}$ disappearance & {$[82,85-87](\pi / \mu$ DIF) } & \\
$3+1+\mathcal{N}$ & {$[10]($ MiniBooNE BNB $\nu)$} & $\mathcal{N}$ \\
\hline \hline
\end{tabular}

$\sin ^{2} 2 \theta_{e e}=4\left(1-\left|U_{e 4}\right|^{2}\right)\left|U_{e 4}\right|^{2}$ (electron flavor disappearance); $\sin ^{2} 2 \theta_{\mu \mu}=4\left(1-\left|U_{\mu 4}\right|^{2}\right)\left|U_{\mu 4}\right|^{2}$ (muon flavor disappearance); and $\sin ^{2} 2 \theta_{\mu e}=4\left|U_{\mu 4}\right|^{2}\left|U_{e 4}\right|^{2}$ (appearance). This implies that the electron and muon flavor disappearance signals must be consistent with the $\nu_{\mu} \rightarrow \nu_{e}$ appearance signal, limiting the range of $\sin ^{2} 2 \theta_{e \mu}$.

This analysis employed the $3+1$ global fitting code described in Ref. [3]. The list of experiments used in the $3+1$-only fit can be found in Supplemental Material [69] Table II. Compared to Ref. [3], we have added new disappearance results from the STEREO experiment [74] and updated PROSPECT results [73]. In this update, we have not included the results from NEUTRINO-4 [92], since the collaboration has not provided an appropriate data release. We have also not included the latest result from IceCube [93,94], which shows a preferred region at 90\% CL compatible with our light sterile neutrino bestfit point, since the collaboration has not provided enough information to reproduce the analysis. To reiterate, the $3+1$-only global fit omitted the MiniBooNE neutrinomode, antineutrino-mode, and off-axis appearance datasets.

The best-fit parameters are $\Delta m^{2}=1.32 \mathrm{eV}^{2}$ and $\sin ^{2} 2 \theta_{e \mu}=$ $6.9 \times 10^{-4}$. In past $3+1$ fits, the tension between the appearance and disappearance datasets [3], as measured using the PG test, has been very high, with a probability of $4 \times 10^{-6}(4.5 \sigma)$ that the data are explained by the same parameters. Without MiniBooNE appearance in the fit, the probability increases to $7 \times 10^{-3}(2.5 \sigma)$. Thus, the tension is, in large part, due to the MiniBooNE appearance dataset, which we hypothesize has the additional component of $\mathcal{N}$-decay, and, hence, poor agreement with $3+1$-only.

\section{MINIBOONE ANALYSIS}

In order to fit MiniBooNE data for $\mathcal{N}$ decay, we wrote a Monte Carlo simulation for the production and decay of $\mathcal{N}$ in the Booster Neutrino Beam in neutrino mode. Two processes were included for production: Dalitz-like $\pi^{0}$ decay and Primakoff upscattering $\nu A \rightarrow \mathcal{N} A$. The latter is by far the dominant $\mathcal{N}$-production mode for $10 \mathrm{MeV}<m_{\mathcal{N}}<$ $1000 \mathrm{MeV}$. Therefore, we neglected the $\pi^{0}$ decay contribution throughout this study. For the Primakoff mode, we generated incident $\nu_{\mu}$ and $\nu_{e}$ events from the MiniBooNE neutrino-mode flux [95]. We then simulated the upscattering rate on both standard upper-continental crust nuclei [96] and the MiniBooNE $\mathrm{CH}_{2}$ detector medium, using Eq. A6 in Ref. [17] to calculate the total interaction rate and momentum transfer. This process produced a sample of right-handpolarized $\mathcal{N}$ events, predominately forward peaked due to the $1 / t^{2}$ dependence of the differential cross section.

Simulated $\mathcal{N}$ which enter the MiniBooNE detector were forced to decay into a photon and a neutrino, taking into account polarization, and weighted by the decay probability. To incorporate the detector efficiency, eff, we performed a linear fit to the reconstructed gamma-ray 
efficiency as a function of true energy [97], eff $=$ $\left(-0.12 \mathrm{GeV}^{-1}\right) * E_{\text {true }}+0.29$, which we used to weight the $\mathcal{N} \rightarrow \nu \gamma$ events. The true energy and angle of the photons were smeared independently according to the resolution given by the MiniBooNE collaboration. More details on the simulation can be found in Appendix A of the Supplemental Material [69].

Ideally one would fit the background-subtracted 2-D distribution of visible energy, $E_{v}$, vs scattering angle, $\theta$, of the MiniBooNE events to the prediction from $3+1+\mathcal{N}-$ decay. However, the systematic errors for this distribution have not been released by MiniBooNE. They are only available for $E_{\nu}^{Q E}$, which is a combination of $E_{v}$ and $\theta$ given by [98]

$E_{\nu}^{Q E}=\frac{2\left(M_{n}-B\right) E_{v}-\left(\left(M_{n}-B\right)^{2}+M_{e}^{2}-M_{p}^{2}\right)}{2\left(\left(M_{n}-B\right)-E_{v}+\sqrt{\left(E_{v}^{2}-M_{e}^{2}\right)} \cos \theta\right)}$,

where $M_{n}, M_{p}$, and $M_{e}$ are the neutron, proton and electron masses, and $B$ is the binding energy of carbon. This formula accurately describes the neutrino energy in the case of twobody charged-current neutrino scattering with no final state interactions, assuming the neutrinos enter the detector along the axis from which $\theta$ is measured. Thus, it is applicable to the oscillation component of the excess. Though $E_{\nu}^{Q E}$ has no physical meaning when applied to the photons from $\mathcal{N}$ decay, the decay kinematics cause most events to be show up at low $E_{\nu}^{Q E}$. We performed a fit to the MiniBooNE excess in $E_{\nu}^{Q E}$ using statistical and systematic uncertainties. We also performed a separate fit to the scattering angle distribution, although only statistical uncertainty is available in this case.

To isolate the decay component, we subtracted from the MiniBooNE excess the predicted contribution of the oscillation component, which was determined from the $3+1$ only global fit without MiniBooNE data. The remaining excess was fit to the model for dipole production, decay, and observation in the detector as described above. Figure 2 shows confidence regions for both fits in $\left\{d, m_{\mathcal{N}}\right\}$ parameter space, computed assuming Wilks' theorem with two degrees of freedom is valid for the test statistic $\chi^{2}(d, m)-$ $\min _{d, m}\left(\chi^{2}(d, m)\right)$ [99]. We found a region of parameter space consistent with both distributions at the 95\% CL near $d=3 \times 10^{-7} \mathrm{GeV}^{-1}$ and $m_{\mathcal{N}}=400 \mathrm{MeV}$.

\section{RESULTS}

Figure 2 shows that the allowed regions from MiniBooNE fits are substantially lower in $d$ than the NOMAD or LSND limits. The overlapping solution is also at substantially higher $m_{\mathcal{N}}$ than kinematically accessible by LSND. Supernova results [17] set limits in a band from approximately $d=10^{-7}$ to $10^{-11} \mathrm{GeV}^{-1}$, which is below the solution we found for MiniBooNE. Thus, our preferred region lies in a window of allowed parameters.

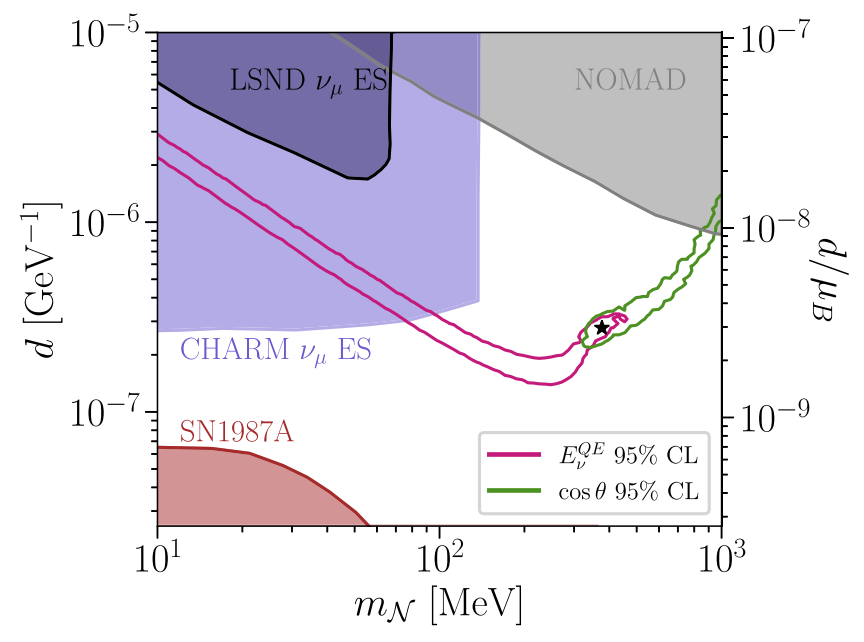

FIG. 2. Preferred regions to explain the MiniBooNE excess in $E_{\nu}^{Q E}$ (pink) and $\cos \theta$ (green) as a function of dipole coupling and $\mathcal{N}$ mass. The black star indicates $\left\{d, m_{\mathcal{N}}\right\}=\left\{2.8 \times 10^{-7} \mathrm{GeV}^{-1}\right.$, $376 \mathrm{MeV}\}$, which lies in the joint $95 \%$ CL allowed region for both distributions. Constraints from other experiments are also shown at the $95 \% \mathrm{CL}$.

We now consider an example HNL decay contribution for $d=2.8 \times 10^{-7} \mathrm{GeV}^{-1}$ and $m_{\mathcal{N}}=376 \mathrm{MeV}$, indicated by the star in Fig. 2. This corresponds to the best fit to the $E_{\nu}^{Q E}$ distribution within the joint $95 \% \mathrm{CL}$ allowed region from the $E_{\nu}^{Q E}$ and $\cos \theta$ fits. Table II shows the $\chi^{2}$ values for the $3+1$ and $3+1+\mathcal{N}$-decay fits to both distributions, indicating significant improvement for the $3+1+\mathcal{N}$ decay model. The global oscillation fit gives tight constraints requiring $\Delta m^{2} \approx 1.32 \mathrm{eV}^{2}$, but allows values of $\sin ^{2} 2 \theta_{\mu e} \in\left[3 \times 10^{-4}, 2 \times 10^{-3}\right]$ at the $90 \%$ CL. The same $\mathcal{N}$ decay fit procedure outlined above has been performed for each end of the allowed $\sin ^{2} 2 \theta_{\mu e}$ range. In each case we again examined the $\left\{d, m_{\mathcal{N}}\right\}$ point that best fits the $E_{\nu}^{Q E}$

TABLE II. $\chi^{2} /$ dof values for $3+1$ and $3+1+\mathcal{N}$-decay models obtained by comparing expectations to the MiniBooNE excess in $E_{\nu}^{Q E}$ and $\cos \theta$. The parameters in column one refer to $\left(\sin ^{2} 2 \theta_{\mu e} \times 10^{-3}, d \times 10^{-7}\left[\mathrm{GeV}^{-1}\right], m_{\mathcal{N}}[\mathrm{MeV}]\right)$. The mass splitting is $1.32 \mathrm{eV}^{2}$ in all cases. The null case (no oscillations and no HNL decay) is also shown in the last row.

\begin{tabular}{lccccc}
\hline \hline & \multicolumn{4}{c}{$\chi^{2} /$ dof } \\
\cline { 2 - 3 } \cline { 5 - 6 } Parameters & \multicolumn{2}{c}{$3+1+\mathcal{N}$} & & \multicolumn{2}{c}{$3+1$} \\
\cline { 2 - 3 } \cline { 5 - 6 }$\left(\sin ^{2} 2 \theta, d, m_{\mathcal{N}}\right)$ & $E_{\nu}^{Q E}$ & $\cos \theta$ & & $E_{\nu}^{Q E}$ & $\cos \theta$ \\
\hline$(0.30,3.1,376)$ & $5.7 / 8$ & $32.1 / 18$ & $30.5 / 10$ & $86.4 / 20$ \\
$(0.69,2.8,376)$ & $7.9 / 8$ & $31.4 / 18$ & & $27.3 / 10$ & $71.8 / 20$ \\
$(2.00,5.6,35)$ & $20.2 / 8$ & $36.7 / 18$ & & $27.6 / 10$ & $40.8 / 20$ \\
$(0,0,0)$ & $34.1 / 10$ & $99.4 / 20$ & & same & same \\
\hline \hline
\end{tabular}



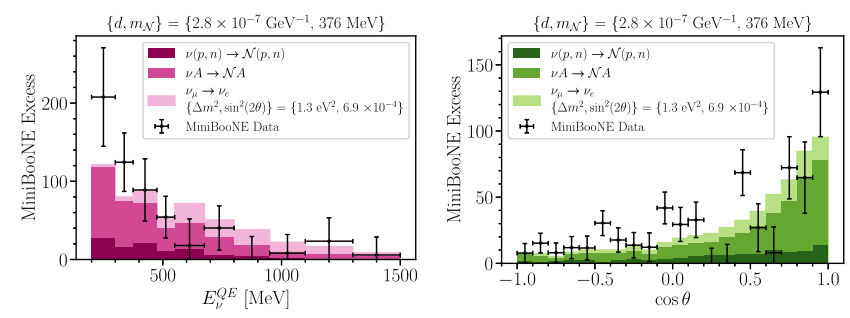

FIG. 3. $E_{\nu}^{Q E}$ (left) and $\cos \theta$ (right) distributions of the MiniBooNE excess for a representative point of the $3+1+\mathcal{N}$-decay model. The error bars on the energy distribution include systematic and statistical errors, while for the angular distribution only statistical errors are included.

distribution within the joint $95 \% \mathrm{CL}$ region. The $\chi^{2}$ values for these fits are also given in Table II, indicating a preference for a smaller oscillation contribution in MiniBooNE in order to explain both the $\cos \theta$ and $E_{\nu}^{Q E}$ distributions via $\mathcal{N} \rightarrow \nu \gamma$. Table II also gives the $\chi^{2}$ values for the null case, with neither $\mathrm{eV}$-scale oscillations nor HNL decay.

Figure 3 presents the MiniBooNE excess in $E_{\nu}^{Q E}$ (left) and $\cos \theta$ (right) compared with the model prediction. This figure includes both the global fit oscillation contribution for $\Delta m^{2}=1.3 \mathrm{eV}^{2}$ and $\sin ^{2} 2 \theta_{e e}=6.9 \times 10^{-7}$, and $\mathrm{HNL}$ decay contribution for $d=2.8 \times 10^{-7} \mathrm{GeV}^{-1}$ and $m_{\mathcal{N}}=$ $376 \mathrm{MeV}$. We reemphasize here that the oscillation contribution shown on these plots comes from a global fit to the $3+1$-only model not including MiniBooNE data. Good agreement is observed for both distributions, especially noting again the lack of systematic errors for the angular distribution, which dominate over statistical errors in the MiniBooNE electronlike analysis [10].

Along with energy and angle, it is essential for the model to be consistent with the recently published timing distribution of the MiniBooNE excess with respect to the proton-beam bunch. The excess events occur within $\pm 4 \mathrm{~ns}$ of the observed $\nu_{\mu}$ events. The time of flight depends on the location at which the HNL is produced. For the preferred $m_{\mathcal{N}} \sim 400 \mathrm{MeV}$, the majority of $\mathcal{N}$ events come from upscattering within the MiniBooNE detector followed by $\mathcal{N}$ decay after travel distances of $\mathcal{O}(50 \mathrm{~cm})$. This leads to timing delays of $<2 \mathrm{~ns}$, well within the MiniBooNE constraint.

\section{CONCLUSION}

We have explored a $3+1+\mathcal{N}$-decay model through fits to the $3+1$-only and MiniBooNE-neutrino-mode datasets. The former yields best-fit oscillation parameters of $\Delta m^{2}=$ $1.3 \mathrm{eV}^{2}$ and $\sin ^{2} 2 \theta_{e \mu}=6.9 \times 10^{-4}$. The latter narrows the HNL mass to be within $300-400 \mathrm{MeV}$ and the dipole coupling strength to be within $2.5-3.5 \times 10^{-7} \mathrm{GeV}^{-1}$. This model produces a $\Delta \chi^{2} /$ dof improvement of $19.3 / 2$
(40.3/2) compared to the global $3+1$ scenario for the fit to the MiniBooNE energy (angular) distribution. This large improvement in $\Delta \chi^{2}$ motivates a more detailed analysis by MiniBooNE. Ideally, the experiment would perform a joint fit to the two-dimensional visible energy and angle distribution, using a full covariance matrix.

Our model also makes very specific predictions for the experiments now running in Fermilab's Short-Baseline Neutrino Program [100]. These experiments make use of liquid-argon time-projection chambers (LArTPCs) that can separate photon showers from electron showers with $\sim 85 \%$ accuracy $[101,102]$. Because the experiments run in the same beamline and are located within $\sim 50 \mathrm{~m}$ of MiniBooNE, the flux is nearly identical. Thus, the ratio of oscillation to HNL decay contributions for the far detectors-MicroBooNE and ICARUS-will be very similar to that of the MiniBooNE case presented here, with $\sim 75 \%$ of the excess events predicted to be single photons. The photon signature will have large backgrounds even in an LArTPC detector, especially from neutral current $\Delta$ baryon production with decays to one or two photons plus a neutron, as well as photons from neutrino interactions produced outside the detector [103]. However, this background rate will be well constrained from reconstructed $\Delta$-decay events with a proton. Also, the energy-angle correlation of the photon in the decay, which depends strongly on $m_{\mathcal{N}}$, can be used for background rejection since this parameter is predicted to have a narrow range of values. The oscillation rates for MicroBooNE and ICARUS are predicted to be low. Within statistics, we predict that no clear oscillation signature will be observed.

Beyond the SBN program, our model can also produce signatures in $\nu_{\mu}$ ES searches at MINER $\nu \mathrm{A}$ and $\mathrm{NO} \nu \mathrm{A}$, as well as dedicated searches for single photons in T2K [104]. Additionally, at neutrino energies of $\mathcal{O}(100 \mathrm{GeV})$ the long decay length produces "double-bang" morphologies in IceCube and CCFR [45,105-107], which would be a smoking gun signature for our model.

In summary, we have presented a new physics model including neutrino-partners with masses of $\mathcal{O}(1 \mathrm{eV})$ that participate in oscillations and $\mathcal{O}(100 \mathrm{MeV})$ that decay to single photons. This model can simultaneously explain the MiniBooNE anomaly and relieve tension in the global experimental picture for $3+1$ oscillations. The results indicate very narrow ranges of HNL decay and oscillation parameters; thus, this is a highly predictive result that can be further tested by existing experiments in the near future.

\section{ACKNOWLEDGMENTS}

M. H. S. is supported by NSF Grant No. PHY-1707971. NSF Grant No. PHY-1801996 supported C. A. A., J. M. C., A. D., and N. W. K. for this work. Additionally, C. A. A. is 
supported by the Faculty of Arts and Sciences of Harvard University. This material is based upon work supported by the National Science Foundation Graduate Research Fellowship under Grant No. 1745302. M. A. U. is supported by the Department of Physics at the University of
Cambridge and S. V. is supported by the STFC. We thank the MiniBooNE Collaboration for useful input, and Gabriel Collin and William Louis for comments on the draft of this paper. Finally, we thank Matheus Hostert and Ryan Plestid for useful discussions.
[1] G. Collin, C. Argüelles, J. Conrad, and M. Shaevitz, Phys. Rev. Lett. 117, 221801 (2016).

[2] M. Dentler, A. Hernández-Cabezudo, J. Kopp, P. A. N. Machado, M. Maltoni, I. Martinez-Soler, and T. Schwetz, J. High Energy Phys. 08 (2018) 010.

[3] A. Diaz, C. Argüelles, G. Collin, J. Conrad, and M. Shaevitz, Phys. Rep. 884, 1 (2020).

[4] S. Böser, C. Buck, C. Giunti, J. Lesgourgues, L. Ludhova, S. Mertens, A. Schukraft, and M. Wurm, Prog. Part. Nucl. Phys. 111, 103736 (2020).

[5] M. Maltoni, T. Schwetz, and J. W. F. Valle, Phys. Rev. D 65, 093004 (2002).

[6] M. Maltoni and T. Schwetz, Phys. Rev. D 68, 033020 (2003).

[7] C. Giunti, M. Laveder, Y. F. Li, and H. W. Long, Phys. Rev. D 88, 073008 (2013).

[8] M. Dentler, I. Esteban, J. Kopp, and P. Machado, Phys. Rev. D 101, 115013 (2020).

[9] A. de Gouvêa, O. Peres, S. Prakash, and G. Stenico, J. High Energy Phys. 07 (2020) 141.

[10] A. A. Aguilar-Arevalo et al. (MiniBooNE Collaboration), Phys. Rev. D 103, 052002 (2021).

[11] S. Gninenko, Phys. Rev. Lett. 103, 241802 (2009).

[12] D. McKeen and M. Pospelov, Phys. Rev. D 82, 113018 (2010).

[13] C. Dib, J. C. Helo, S. Kovalenko, and I. Schmidt, Phys. Rev. D 84, 071301 (2011).

[14] S. Gninenko, Phys. Lett. B 710, 86 (2012).

[15] M. Masip, P. Masjuan, and D. Meloni, J. High Energy Phys. 01 (2013) 106.

[16] P. Ballett, S. Pascoli, and M. Ross-Lonergan, J. High Energy Phys. 04 (2017) 102.

[17] G. Magill, R. Plestid, M. Pospelov, and Y.-D. Tsai, Phys. Rev. D 98, 115015 (2018).

[18] O. Fischer, A. Hernández-Cabezudo, and T. Schwetz, Phys. Rev. D 101, 075045 (2020).

[19] E. Bertuzzo, S. Jana, P. A. N. Machado, and R. Zukanovich Funchal, Phys. Lett. B 791, 210 (2019).

[20] E. Bertuzzo, S. Jana, P. A. N. Machado, and R. Zukanovich Funchal, Phys. Rev. Lett. 121, 241801 (2018).

[21] P. Ballett, S. Pascoli, and M. Ross-Lonergan, Phys. Rev. D 99, 071701 (2019).

[22] C. A. Argüelles, M. Hostert, and Y.-D. Tsai, Phys. Rev. Lett. 123, 261801 (2019).

[23] P. Ballett, M. Hostert, and S. Pascoli, Phys. Rev. D 99, 091701 (2019).
[24] P. Ballett, M. Hostert, and S. Pascoli, Phys. Rev. D 101, 115025 (2020).

[25] A. Abdullahi, M. Hostert, and S. Pascoli, Phys. Lett. B 820, 136531 (2021).

[26] W. Abdallah, R. Gandhi, and S. Roy, Phys. Rev. D 104, 055028 (2021).

[27] W. Abdallah, R. Gandhi, and S. Roy, J. High Energy Phys. 12 (2020) 188.

[28] M. Drewes et al., J. Cosmol. Astropart. Phys. 01 (2017) 025.

[29] A. Abada, K. N. Abazajian, A. Abdullahi, S. K. Agarwalla, J. A. Aguilar, W. Altmannshofer, C. A. Argüelles, A. B. Balantekin, G. Barenboim, B. Batell et al., Opportunities and signatures of non-minimal heavy neutral leptons, Letter of Interest submitted to the Snowmass2021, https://www.snowmass21.org/docs/files/summaries/NF/ SNOWMASS21-NF2_NF3-EF9_EF0-RF4_RF6-CF1_ CF0-TF8_TF11_Matheus_Hostert-041.pdf (2020).

[30] K. Fujikawa and R. Shrock, Phys. Rev. Lett. 45, 963 (1980).

[31] P. B. Pal and L. Wolfenstein, Phys. Rev. D 25, 766 (1982).

[32] R. E. Shrock, Nucl. Phys. B206, 359 (1982).

[33] M. Dvornikov and A. Studenikin, Phys. Rev. D 69, 073001 (2004).

[34] C. Giunti and A. Studenikin, Rev. Mod. Phys. 87, 531 (2015).

[35] V. Brdar, A. Greljo, J. Kopp, and T. Opferkuch, J. Cosmol. Astropart. Phys. 01 (2021) 039, see arXiv version v3 for updated constraint plot summary.

[36] J. F. Nieves, Phys. Rev. D 28, 1664 (1983).

[37] K. N. Abazajian, Phys. Rep. 711-712, 1 (2017).

[38] M. Tanabashi et al. (Particle Data Group Collaboration), Phys. Rev. D 98, 030001 (2018).

[39] I. Esteban, M. Gonzalez-Garcia, A. Hernandez-Cabezudo, M. Maltoni, and T. Schwetz, J. High Energy Phys. 01 (2019) 106.

[40] P. de Salas, D. Forero, C. Ternes, M. Tortola, and J. W. F. Valle, Phys. Lett. B 782, 633 (2018).

[41] F. Capozzi, E. Lisi, A. Marrone, D. Montanino, and A. Palazzo, Nucl. Phys. B908, 218 (2016).

[42] J. A. Formaggio, J. M. Conrad, M. Shaevitz, A. Vaitaitis, and R. Drucker, Phys. Rev. D 57, 7037 (1998).

[43] A. Baha Balantekin and B. Kayser, Annu. Rev. Nucl. Part. Sci. 68, 313 (2018).

[44] A. de Gouvea, P. J. Fox, B. J. Kayser, and K. J. Kelly, Phys. Rev. D 104, 015038 (2021). 
[45] P. Coloma, P. A. N. Machado, I. Martinez-Soler, and I. M. Shoemaker, Phys. Rev. Lett. 119, 201804 (2017).

[46] S. Hannestad, I. Tamborra, and T. Tram, J. Cosmol. Astropart. Phys. 07 (2012) 025.

[47] M. Lattanzi and M. Gerbino, Front. Phys. 5, 70 (2018).

[48] A. M. Knee, D. Contreras, and D. Scott, J. Cosmol. Astropart. Phys. 07 (2019) 039.

[49] J. M. Berryman, Phys. Rev. D 100, 023540 (2019).

[50] S. Gariazzo, P. F. de Salas, and S. Pastor, J. Cosmol. Astropart. Phys. 07 (2019) 014.

[51] S. Hagstotz, P. F. de Salas, S. Gariazzo, M. Gerbino, M. Lattanzi, S. Vagnozzi, K. Freese, and S. Pastor, arXiv: 2003.02289.

[52] M. Adams, F. Bezrukov, J. Elvin-Poole, J. J. Evans, P. Guzowski, B. O. Fearraigh, and S. Söldner-Rembold, Eur. Phys. J. C 80, 758 (2020).

[53] G. Gelmini, S. Palomares-Ruiz, and S. Pascoli, Phys. Rev. Lett. 93, 081302 (2004).

[54] J. Hamann, S. Hannestad, G. G. Raffelt, and Y. Y. Y. Wong, J. Cosmol. Astropart. Phys. 09 (2011) 034.

[55] S. Hannestad, R. S. Hansen, and T. Tram, Phys. Rev. Lett. 112, 031802 (2014).

[56] B. Dasgupta and J. Kopp, Phys. Rev. Lett. 112, 031803 (2014).

[57] M. Archidiacono, S. Hannestad, R. S. Hansen, and T. Tram, Phys. Rev. D 91, 065021 (2015).

[58] M. Archidiacono, S. Hannestad, R. S. Hansen, and T. Tram, Phys. Rev. D 93, 045004 (2016).

[59] N. Saviano, O. Pisanti, G. Mangano, and A. Mirizzi, Phys. Rev. D 90, 113009 (2014).

[60] X. Chu, B. Dasgupta, and J. Kopp, J. Cosmol. Astropart. Phys. 10 (2015) 011.

[61] J. F. Cherry, A. Friedland, and I. M. Shoemaker, arXiv: 1605.06506.

[62] X. Chu, B. Dasgupta, M. Dentler, J. Kopp, and N. Saviano, J. Cosmol. Astropart. Phys. 11 (2018) 049.

[63] N. Song, M. C. Gonzalez-Garcia, and J. Salvado, J. Cosmol. Astropart. Phys. 10 (2018) 055.

[64] Y. Farzan, Phys. Lett. B 797, 134911 (2019).

[65] J. M. Cline, Phys. Lett. B 802, 135182 (2020).

[66] B. Shakyaand J. D. Wells, J. High Energy Phys. 02 (2019) 174.

[67] M. Escudero, J. Lopez-Pavon, N. Rius, and S. Sandner, J. High Energy Phys. 12 (2020) 119.

[68] M. Berbig, S. Jana, and A. Trautner, Phys. Rev. D 102, 115008 (2020).

[69] Please see Supplemental Material at http://link.aps.org/ supplemental/10.1103/PhysRevD.104.095005 for more details on both the MiniBooNE MeV-scale HNL dipole model simulation and the global fit for the $\mathrm{eV}$-scale sterile neutrino oscillation parameters.

[70] Y. Declais et al., Nucl. Phys. B434, 503 (1995).

[71] Y. J. Ko et al. (NEOS Collaboration), Phys. Rev. Lett. 118, 121802 (2017).

[72] I. Alekseev et al. (DANSS Collaboration), Phys. Lett. B 787, 56 (2018).

[73] M. Andriamirado, A. B. Balantekin, H. R. Band, C. D. Bass, D. E. Bergeron, D. Berish, N. S. Bowden, J. P. Brodsky, C. D. Bryan, T. Classen et al. (PROSPECT Collaboration), Phys. Rev. D 103, 032001 (2021).
[74] H. Almazán, L. Bernard, A. Blanchet, A. Bonhomme, C. Buck, P. del Amo Sanchez, I. El Atmani, J. Haser, F. Kandzia, S. Kox et al. (STEREO Collaboration), Phys. Rev. D 102, 052002 (2020).

[75] J. M. Conrad and M. H. Shaevitz, Phys. Rev. D 85, 013017 (2012).

[76] F. Kaether, W. Hampel, G. Heusser, J. Kiko, and T. Kirsten, Phys. Lett. B 685, 47 (2010).

[77] J. N. Abdurashitov et al. (SAGE Collaboration), Phys. Rev. C 80, 015807 (2009).

[78] A. Aguilar-Arevalo et al. (LSND Collaboration), Phys. Rev. D 64, 112007 (2001).

[79] B. Armbruster et al. (KARMEN Collaboration), Phys. Rev. D 65, 112001 (2002).

[80] P. Astier et al. (NOMAD Collaboration), Phys. Lett. B 570, 19 (2003).

[81] G. Cheng et al. (MiniBooNE, SciBooNE Collaborations), Phys. Rev. D 86, 052009 (2012).

[82] I. E. Stockdale et al., Phys. Rev. Lett. 52, 1384 (1984).

[83] P. Adamson et al. (MINOS Collaboration), Phys. Rev. Lett. 108, 191801 (2012).

[84] P. Adamson et al. (MINOS Collaboration), Phys. Rev. D 84, 071103 (2011).

[85] K. B. M. Mahn et al. (SciBooNE, MiniBooNE Collaborations), Phys. Rev. D 85, 032007 (2012).

[86] F. Dydak et al., Phys. Lett. 134B, 281 (1984).

[87] P. Adamson et al. (MINOS Collaboration), Phys. Rev. Lett. 117, 151803 (2016).

[88] A. A. Aguilar-Arevalo et al. (MiniBooNE Collaboration), Phys. Rev. Lett. 98, 231801 (2007).

[89] A. A. Aguilar-Arevalo et al. (MiniBooNE Collaboration), Phys. Rev. Lett. 110, 161801 (2013).

[90] P. Adamson et al. (MiniBooNE, MINOS Collaborations), Phys. Rev. Lett. 102, 211801 (2009).

[91] K. Abe et al. (T2K Collaboration), Phys. Rev. D 100, 052006 (2019).

[92] A. P. Serebrov et al. (NEUTRINO-4 Collaboration), Pis'ma Zh. Eksp. Teor. Fiz. 109, 209 (2019).

[93] M. Aartsen et al. (IceCube Collaboration), Phys. Rev. Lett. 125, 141801 (2020).

[94] M. Aartsen et al. (IceCube Collaboration), Phys. Rev. D 102, 052009 (2020).

[95] A. A. Aguilar-Arevalo, C. E. Anderson, A. O. Bazarko, S. J. Brice, B. C. Brown, L. Bugel, J. Cao, L. Coney, J. M. Conrad, D. C. Cox et al., Phys. Rev. D 79 (2009).

[96] F. W. Clarke and H.S. Washington, Technical Report, 1924, http://pubs.er.usgs.gov/publication/pp127.

[97] Z. Pavlovic, R. Van de Water, and S. Zeller, Technical Report, 2012, https://rtayloe.pages.iu.edu/MB/data-release/ nue_nuebar_2012/efficiency/MB_nu_nubar_combined_ release.html.

[98] A. A. Aguilar-Arevalo, B. C. Brown, J. M. Conrad, R. Dharmapalan, A. Diaz, Z. Djurcic, D. A. Finley, R. Ford, G. T. Garvey, S. Gollapinni et al. MiniBooNE Collaboration), Phys. Rev. D 103, 052002 (2021).

[99] S. S. Wilks, Ann. Math. Stat. 9, 60 (1938).

[100] M. Antonello et al. (MicroBooNE, LAr1-ND, ICARUSWA104 Collaborations), arXiv:1503.01520. 
[101] C. Adams, M. Alrashed, R. An, J. Anthony, J. Asaadi, A. Ashkenazi, S. Balasubramanian, B. Baller, C. Barnes, G. Barr et al., J. Instrum. 15, P02007P02007 (2020).

[102] R. Acciarri, C. Adams, J. Asaadi, B. Baller, T. Bolton, C. Bromberg, F. Cavanna, E. Church, D. Edmunds, A. Ereditato et al. (ArgoNeuT Collaboration), Phys. Rev. D 95, 072005 (2017).

[103] MicroBooNE Collaboration (2020), https://microboone .fnal.gov/wp-content/uploads/MICROBOONE-NOTE1087-PUB.pdf.
[104] K. Abe et al. (T2K Collaboration), J. Phys. G 46, 08LT01 (2019).

[105] P. Coloma, Eur. Phys. J. C 79, 748 (2019).

[106] P. de Barbaro et al. (CCFR Collaboration), in Proceedings of the 26th International Conference on High-energy Physics (1992), https://inspirehep.net/conferences/967647 ?ui-citation-summary=true.

[107] H. S. Budd et al. (CCFR Collaboration), in Beyond the Standard Model III (Note change of dates from Jun 8-10) (1992), https://lib-extopc.kek.jp/preprints/PDF/ 1992/9212/9212531.pdf. 